\title{
Swallow Evaluation
}

National Cancer Institute

\section{Source}

National Cancer Institute. Swallow Evaluation. NCI Thesaurus. Code C48874.

The administration of liquids in assorted thicknesses to determine the ability of a patient to swallow without aspiration. Swallow evaluation involves a variety of approaches, but may be divided into endoscopic and non-endoscopic techniques. 